\title{
Inversed linear dichroism in F K-edge NEXAFS spectra of fluorinated planar aromatic molecules
}

de Oteyza, D. G.; Sakko, A.; El-Sayed, A.; Goiri, E.; Floreano, L.; Cossaro, A.; García Lastra, Juan Maria; Rubio, A.; Ortega, J. E.

Published in:

Physical Review B Condensed Matter

Link to article, DOI:

10.1103/PhysRevB.86.075469

Publication date:

2012

Document Version

Publisher's PDF, also known as Version of record

Link back to DTU Orbit

Citation $(A P A)$ :

de Oteyza, D. G., Sakko, A., El-Sayed, A., Goiri, E., Floreano, L., Cossaro, A., García Lastra, J. M., Rubio, A., \& Ortega, J. E. (2012). Inversed linear dichroism in F K-edge NEXAFS spectra of fluorinated planar aromatic molecules. Physical Review B Condensed Matter, 86(7), 075469. https://doi.org/10.1103/PhysRevB.86.075469

\section{General rights}

Copyright and moral rights for the publications made accessible in the public portal are retained by the authors and/or other copyright owners and it is a condition of accessing publications that users recognise and abide by the legal requirements associated with these rights.

- Users may download and print one copy of any publication from the public portal for the purpose of private study or research.

- You may not further distribute the material or use it for any profit-making activity or commercial gain

- You may freely distribute the URL identifying the publication in the public portal 


\title{
Inversed linear dichroism in $\mathbf{F} \boldsymbol{K}$-edge NEXAFS spectra of fluorinated planar aromatic molecules
}

\author{
D. G. de Oteyza, ${ }^{1,2, *}$ A. Sakko, ${ }^{3,4}$ A. El-Sayed, ${ }^{5}$ E. Goiri, ${ }^{6}$ L. Floreano, ${ }^{7}$ A. Cossaro, ${ }^{7}$ J. M. Garcia-Lastra, ${ }^{8,9}$ \\ A. Rubio, ${ }^{1,6,9,10}$ and J. E. Ortega ${ }^{1,5,6}$ \\ ${ }^{1}$ Centro de Física de Materiales CSIC-UPVIEHU, Materials Physics Center (MPC), Paseo Manuel Lardizabal 3, 20018 San Sebastián, Spain \\ ${ }^{2}$ University of California at Berkeley, Physics Department, Berkeley, California 94720, USA \\ ${ }^{3}$ Department of Applied Physics, Aalto University, P.O. Box 11100, 00076 Aalto, Finland \\ ${ }^{4}$ Department of Physics, P.O. Box 64, FI-00014, University of Helsinki, Finland \\ ${ }^{5}$ Departamento de Física Aplicada, Universidad del Pais Vasco, Pza. Oñate 2, 20018 San Sebastián, Spain \\ ${ }^{6}$ Donostia International Physics Center, Paseo Manuel Lardizabal 4, 20018 San Sebastián, Spain \\ ${ }^{7}$ CNR-IOM, Instituto Officina dei Materiali, Laboratorio TASC, Bassovizza SS-14, km 163.5, I-34149 Trieste, Italy \\ ${ }^{8}$ Center for Atomic-Scale Materials Design (CAMD), Department of Physics, Technical University of Denmark. \\ Fysikvej 1, 2800 Kgs. Lyngby, Denmark \\ ${ }^{9}$ Theoretical Spectroscopy Facility (ETSF), and Nano-Bio Spectroscopy Group, ETSF Scientific Development Centre, \\ Universidad del País Vasco, Av. Tolosa 72, E-20018 San Sebastián, Spain \\ ${ }^{10}$ Fritz-Haber-Institut der Max-Planck-Gesellschaft, Theory Department, Faradayweg 4-6, D-14195 Berlin-Dahlem, Germany
}

(Received 31 May 2012; published 29 August 2012)

\begin{abstract}
The symmetry and energy distribution of unoccupied molecular orbitals is addressed in this work by means of NEXAFS and density functional theory calculations for planar, fluorinated organic semiconductors (perfluorinated copper phthalocyanines and perfluoropentacene). We demonstrate how molecular orbitals with significant density of states on the fluorine atoms show different symmetry from those mainly located on $\mathrm{C}$ and $\mathrm{N}$ atoms. As a result, the angle-dependent linear dichroism in NEXAFS F $K$-edge spectra is inversed with respect to that in the $\mathrm{C}$ and $\mathrm{N} K$-edges. In addition, the significant overlap in energy of $\pi^{*}$ and $\sigma^{*}$ orbitals throughout the $\mathrm{F} K$-edge spectrum hampers its use for analysis of molecular orientations from angle-dependent NEXAFS measurements.
\end{abstract}

DOI: 10.1103/PhysRevB.86.075469

PACS number(s): 61.05.cj

\section{INTRODUCTION}

Interfaces between organic semiconducting thin films and inorganic substrates are not only key to the performance of many devices but also serve as model systems for fundamental studies. ${ }^{1}$ Many semiconducting oligomers, and in particular those on which we focus in this article (Fig. 1), are planar, with their $\pi$-orbitals sticking out perpendicular to the molecular plane. Due to this pronounced structural anisotropy, which is, in turn, transferred to their optoelectronic properties, the molecular orientation at the interfaces and within the films is of utmost importance for their response in optoelectronic devices. By way of example, charge transport within molecular films is fastest along the maximized intermolecular $\pi$-orbital overlap direction; ${ }^{1,2}$ charge transfer at metal-organic interfaces is commonly favored for frontier $\pi$-orbitals facing the surface,,$^{1,2}$ or from a growth point of view, the first layers in contact with the metal can seed the self-assembly of subsequent layers with a specific orientation via anisotropic intermolecular interactions. ${ }^{3,4}$ A selected number of experimental techniques is able to access such critical information on molecular orientation in thin films, among which one of the most popular is near-edge $\mathrm{x}$-ray absorption spectroscopy (NEXAFS).

NEXAFS is a widely used technique providing useful information on the electronic and also crystalline structure of the samples. ${ }^{5}$ Most NEXAFS studies on the characterization of molecular arrangements in thin films of organic materials are focused on the C $K$-edge. ${ }^{6-8}$ However, the presence of heteroatoms in molecules can also be used to gain additional information on spatially defined alternative sites. ${ }^{9-12}$ We show in this article that careful consideration of the molecular electronic orbitals has to be taken before drawing conclusions on the molecular arrangement, since NEXAFS spectra from different heteroatoms can show an inversed linear dichroism behavior with respect to that of $\mathrm{C}$. This is the case of $\mathrm{F} K$-edge NEXAFS spectra, as we show for two different perfluorinated oligomers. In particular, we have measured various NEXAFS $K$-edge spectra on perfluorinated copper phthalocyanines $\left(\mathrm{F}_{16} \mathrm{CuPc}\right)$ and perfluoropentacene (PFP), two relevant molecules as air-stable $n$-type organic semiconductors. ${ }^{13}$ Their respective molecular structures are depicted in Fig. 1(a), where it can be seen that both molecules contain $\mathrm{C}$ and $\mathrm{F}$ atoms and additional $\mathrm{N}$ and $\mathrm{Cu}$ in the case of $\mathrm{F}_{16} \mathrm{CuPc}$.

\section{METHODS}

Core-level spectroscopy and NEXAFS measurements were performed at the beamline ALOISA of the synchrotron light source ELETTRA in Trieste (Italy). Prior to evaporation, the surface was checked for its cleanliness and order by x-ray photoelectron spectroscopy (XPS) and reflection high-energy electron diffraction (RHEED). The sample coverage was monitored by means of a quartz crystal microbalance, assuming a density of $2.05 \mathrm{~g} / \mathrm{cm}^{3}$ for $\mathrm{F}_{16} \mathrm{CuPc}^{14}$ and $2.15 \mathrm{~g} / \mathrm{cm}^{3}$ for PFP ${ }^{15}$ and further cross-checked with an analysis of the $\mathrm{Au} 4 f$ and $\mathrm{C} 1 s$ core-level intensities.

The manipulator is coaxial with the photon beam, which allows changing the orientation of the surface with respect to linear polarization of the beam, while keeping constant the grazing angle at $4^{\circ}$, i.e., without changing the beam footprint on the sample. For more details about the scattering geometry, see Ref. 32. Under such conditions, the intensities from NEXAFS spectra at different polarizations can be directly 

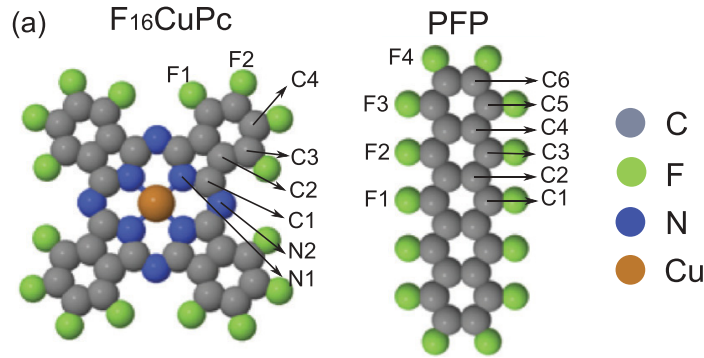

(b)

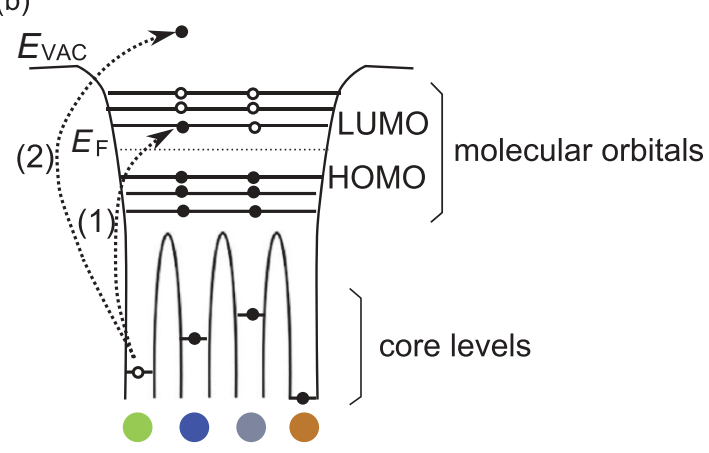

FIG. 1. (Color online) (a) Molecular structures of perfluorinated copper phthalocyanine $\left(\mathrm{F}_{16} \mathrm{CuPc}\right)$ and PFP. (b) Schematic representation of electronic levels in a molecule, including localized core levels, as well as delocalized molecular orbitals. The experimental techniques used in this work include XPS and NEXAFS. In the former, a core level is excited by fixed energy photons above the vacuum level $E_{\mathrm{vac}}$ (excitation 2), and the distribution of kinetic energies are measured. In the latter, the photon energy is scanned in the range of transitions from core levels to empty molecular orbitals (excitation 1). The decay of such excited states causes the emission of secondary electrons that are then measured as a function of the photon energy.

compared with one another. Artifacts in the relative intensities of different polarizations due to varying molecular coverage upon beam drift on samples with heterogeneous coverage can be excluded by repeated measurements on different samples, and by the similar postedge intensities measured, that is, intensity at energies significantly higher than the resonances. In the case of the unchanged beam footprint, it is proportional to the sample coverage (see Supplemental Material ${ }^{16}$ ).

NEXAFS spectra were taken in partial electron yield. Spectra measured at the $\mathrm{C}$ and $\mathrm{N} K$-edges have been calibrated by acquisition of the $1 s-\pi^{*}$ gas phase transitions of $\mathrm{CO}$ and $\mathrm{N}_{2}$ at $h v=287.4 \mathrm{eV}$ and $h v=401.1 \mathrm{eV}$, respectively. ${ }^{17}$ In the absence of a reference signal for the absolute photon energy calibration in the F $K$-edge spectra, we have alternatively calibrated it by the $\mathrm{Fe}$ absorption line (set to $706.8 \mathrm{eV})^{18}$ observed in the drain current taken on the multilayer metal coating of the last mirror of the beamline. The photoemission data shown in Fig. 2 for $\mathrm{F}_{16} \mathrm{CuPc}$ were taken with excitation energies of $395 \mathrm{eV}$ for the $\mathrm{C}, 650$ for the $\mathrm{N}$, and $795 \mathrm{eV}$ for the F core levels, with pass energies of $21.25 \mathrm{eV}$. For PFP, photon energies and pass energies were, respectively, $795 \mathrm{eV}$ and $21.25 \mathrm{eV}$ for $\mathrm{F}$ core levels and $400 \mathrm{eV}$ and $10.625 \mathrm{eV}$ for $\mathrm{C}$ core levels. The spectrometer resolution is $1 \%$ of the pass energy. Voigt peaks and Shirley background were used for the spectral fitting.

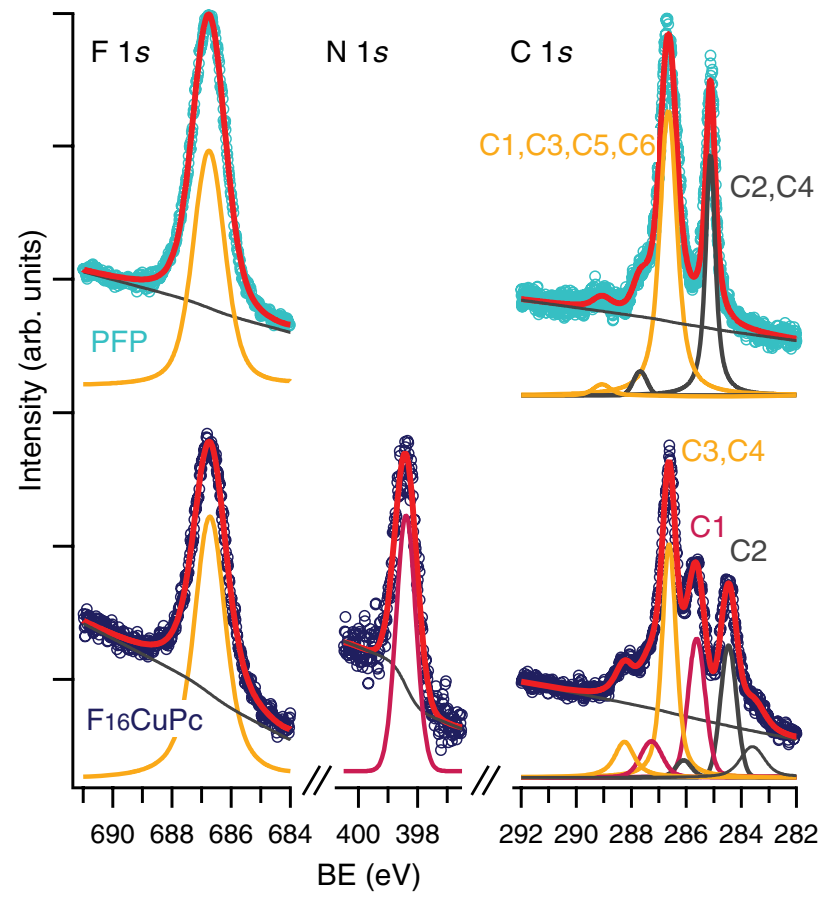

FIG. 2. (Color online) Characteristic XPS spectra for $\mathrm{F}_{16} \mathrm{CuPc}$ and PFP monolayers on $\mathrm{Au}(111)$. The observed core levels correspond to the initial states for the electron transitions of our subsequently measured NEXAFS spectra. The specific atoms giving rise to the various components in the $\mathrm{C} 1 s$ spectra are labeled following the nomenclature of Fig. 1. The SU satellites are color coded according to the main peaks.

Calculations of NEXAFS spectra have been performed with StoBe-deMon code. ${ }^{19}$ The exchange-correlation energy has been computed within the local density approximation (LDA) with the Vosko-Wilk-Nusair functional. ${ }^{20}$ The structures of $\mathrm{F}_{16} \mathrm{CuPc}$ and PFP have been relaxed until a maximum force below $0.03 \mathrm{eV} / \AA$ was obtained. The spectra were calculated within the framework presented in Ref. 34, using the transition potential approximation (TPA). ${ }^{21}$ Triple- $\zeta$ plus valence polarization-type basis sets were used for all the nonexcited atoms. For the excited site, we used the IGLO-III basis set together with a set of diffuse basis functions. ${ }^{22}$ They improve the description of the orbital relaxation effects and the modeling of the delocalized unoccupied states, as described in Ref. 21 and in references therein. To create a welllocalized core orbital, we used the model core potential method (MCP). ${ }^{23}$ With MCP, the nucleus and the inner-shell electrons of all the carbon atoms except the excited one are replaced by model potentials. This ensures the localization of the initial $1 \mathrm{~s}$ orbital. In this work separate calculations were performed for all nonequivalent excited sites (four carbon, two fluorine, and two nitrogen nonequivalent atoms in $\mathrm{F}_{16} \mathrm{CuPc}$ and six carbon and four fluorine nonequivalent atoms in PFP), and the final spectra were obtained as appropriately weighted averages of these calculations. We have repeated the calculations within the general gradient approximation (GGA) with the PerdewBurke-Ernzerhof (PBE) functional. ${ }^{24}$ LDA and GGA results are very similar, apart from a rigid energy shift. 


\section{RESULTS AND DISCUSSION}

In a NEXAFS experiment, the samples are illuminated with photons whose energy is scanned across transitions from core levels to empty orbitals, which, in the case of molecules, start with the so-called lowest unoccupied molecular orbital (LUMO; Fig. 1). Thereby generated excited states subsequently decay, causing the emission of photons via photoluminescence, or of secondary electrons, both of which can then be measured as a function of the photon energy. Spectral resonances therefore correspond to the difference in energy between a core level and the LUMO or higher unoccupied electronic levels. However, because the excited electron remains in the sample, its coulomb interaction with the associated hole [exciton binding energy (BE)] reduces the apparent energy difference with respect to that in the molecular ground state. ${ }^{5}$ The structural information available from NEXAFS measurements has its origin in the transition matrix elements defining the X-ray absorption cross section. ${ }^{5}$ In the dipole approximation, valid for x-ray wavelengths larger than the $K$-shell diameter and therefore applicable to all our measurements shown in this work, the intensity is proportional to $I \propto|\boldsymbol{e} \cdot\langle f|\boldsymbol{p}| i\rangle|^{2}$, where $\boldsymbol{e}$ is the electric field vector, $\boldsymbol{p}$ is the linear momentum operator of the electrons, $\langle f|$ is the final state, and $|i\rangle$ the initial state. For $K$-shell excitations the initial $1 s$ state is spherically symmetric. The dipole selection rule requires the final state to be a $p$ state, and the vector matrix element $\langle f|\boldsymbol{p}| i\rangle$ points in the direction of that orbital. The measured NEXAFS intensity is maximized when $\boldsymbol{e}$ points along that same direction and shows $a \cos ^{2} \delta$ dependence on the angle $\delta$ between $\boldsymbol{e}$ and the direction of maximum molecular orbital amplitude. Molecular orbitals have a strong directional character directly correlated with the molecular geometry. Such orbital directionality, in combination with polarized x-rays, leads to a pronounced dependence of the absorption with the relative angle between polarization vector and involved orbitals. As a result, $K$-edge spectra measured with linearly polarized light on planar molecules, like those used in this work (Fig. 1), lead to enhanced $\pi^{*}\left(\sigma^{*}\right)$ resonances when the polarization vector is aligned perpendicular (parallel) to the molecular plane. Analysis of the resonance intensities as a function of the relative angle between the substrate surface and $\mathrm{x}$-ray polarization vector thus provides quantitative information of the molecular orientations on the substrate. ${ }^{4,5}$

In a first step we have measured the core-level photoemission, summarized in Fig. 2 for $\mathrm{F}_{16} \mathrm{CuPc}$ and PFP monolayers deposited on $\mathrm{Au}(111)$. These correspond to the initial states in the subsequently measured $K$-edge NEXAFS spectra. If only one core level is present, the NEXAFS resonances can be directly attributed to transitions into successive unoccupied molecular orbitals. In the case of multiple, energetically different core levels, a superposition of transitions from each core level occurs, complicating a direct understanding of the NEXAFS resonances. This is the case of the $\mathrm{C} K$-edge NEXAFS spectra of most organic molecules. In our particular case, $\mathrm{F}_{16} \mathrm{CuPc}$ has four chemically different $\mathrm{C}$ atoms, as labeled in Fig. 1(a). However, only three different peaks are actually resolved, which, starting from the lowest BE, correspond to the $\mathrm{C}$ atoms bonded solely to other $\mathrm{C}$ atoms (C2), bonded to $\mathrm{N}(\mathrm{C} 1)$, and bonded to $\mathrm{F}(\mathrm{C} 3, \mathrm{C} 4)$. As extensively described in previous studies, the shifts to higher BEs with respect to $\mathrm{C} 2$ are directly related to the electron transfer from the $\mathrm{C}$ to the $\mathrm{N}$ and $\mathrm{F}$ atoms, respectively. ${ }^{25,26}$ A similar scenario is observed for PFP, which holds six chemically different $\mathrm{C}$ atoms [Fig. 1(a)], while only two peaks are observed that cumulate the atoms with $(\mathrm{C} 1, \mathrm{C} 3, \mathrm{C} 5, \mathrm{C} 6)$ or without $(\mathrm{C} 2, \mathrm{C} 4)$ bonds to fluorine (Fig. 2). The larger width of the former can be ascribed to the larger number of chemically different atoms included in the signal. Moving on to $\mathrm{F}$, we find two and four chemically different $\mathrm{F}$ atoms on $\mathrm{F}_{16} \mathrm{CuPc}$ and $\mathrm{PFP}$, respectively. $\mathrm{F}_{16} \mathrm{CuPc}$ also holds two different $\mathrm{N}$ atoms. However, the use of multiple, slightly offset components to fit those core-level spectra leads only to marginally better fits, and the use of a single broader peak results in equally satisfactory fits for all $\mathrm{F}$ and $\mathrm{N}$ core levels. In addition to the main peaks, first-order shake-up (SU) satellites, related to simultaneous highest occupied molecular orbital (HOMO)-LUMO excitations by the emitted electrons, had to be included to obtain adequate fits in the case of $\mathrm{C} 1 s$ spectra, while not so for $\mathrm{F} 1 s$ and $\mathrm{N} 1 s$ spectra. The SU satellites showed up consistently shifted by $1.63 \mathrm{eV}$ to higher BEs in $\mathrm{F}_{16} \mathrm{CuPc}$ and by $2.49 \mathrm{eV}$ in PFP, evidencing their respective optical HOMO-LUMO gaps. ${ }^{27}$ Summary of the peak widths ${ }^{28}$ and positions are provided in Table I.

The final states for $K$-edge spectra correspond to the unoccupied $p_{z}$ or $p_{x}-p_{y}$ orbitals. The local density of states (LDOS) from such unoccupied molecular orbitals projected onto the $\mathrm{F}_{16} \mathrm{CuPc}$ and $\mathrm{PFP} \mathrm{C}, \mathrm{N}$, and $\mathrm{F}$ atoms, respectively, is given in Fig. 3 for molecules in gas phase. Molecular orbitals in a core-excited state as in a NEXAFS experiment are expected to be somewhat different from those in the ground state. However, as we will see below, the LDOS from the ground-state calculations still provides adequate qualitative understanding of the linear dichroism found in NEXAFS experiments. For $\mathrm{C}$ atoms, regardless of the molecule, we find that the LDOS at the lowest energies clearly stems solely from $p_{z}$ states. These states are oriented out of the molecular plane and correspond to $\pi^{*}$ orbitals. We observe the first contribution to the LDOS from $p_{x}-p_{y}$ states only at energies well above those of the first $p_{z}$ states. The

TABLE I. BEs and full width at half maximum (FWHM) of the main and SU components in XPS spectra.

\begin{tabular}{|c|c|c|c|c|c|c|c|c|}
\hline & \multicolumn{3}{|c|}{ PFP } & \multicolumn{5}{|c|}{$\mathrm{F}_{16} \mathrm{CuPC}$} \\
\hline & $\mathrm{C} 2, \mathrm{C} 4$ & $\mathrm{C} 1, \mathrm{C} 3, \mathrm{C} 5, \mathrm{C} 6$ & $\mathrm{~F}$ & $\mathrm{C} 2$ & $\mathrm{C} 1$ & $\mathrm{C} 3, \mathrm{C} 4$ & $\mathrm{~N}$ & $\mathrm{~F}$ \\
\hline BEs $(e V)$ & $\begin{array}{c}285.12 \\
(\mathrm{SU} 287.68)\end{array}$ & $\begin{array}{c}286.65 \\
\text { (SU 289.07) }\end{array}$ & 686.75 & $\begin{array}{c}284.47 \\
(\mathrm{SU} 286.1)\end{array}$ & $\begin{array}{c}285.63 \\
\text { (SU 287.27) }\end{array}$ & $\begin{array}{c}286.63 \\
\text { (SU 288.25) }\end{array}$ & 398.39 & 686.72 \\
\hline FWHM (eV) & 0.44 & 0.75 & 1.19 & 0.67 & 0.68 & 0.58 & 0.77 & 1.27 \\
\hline
\end{tabular}



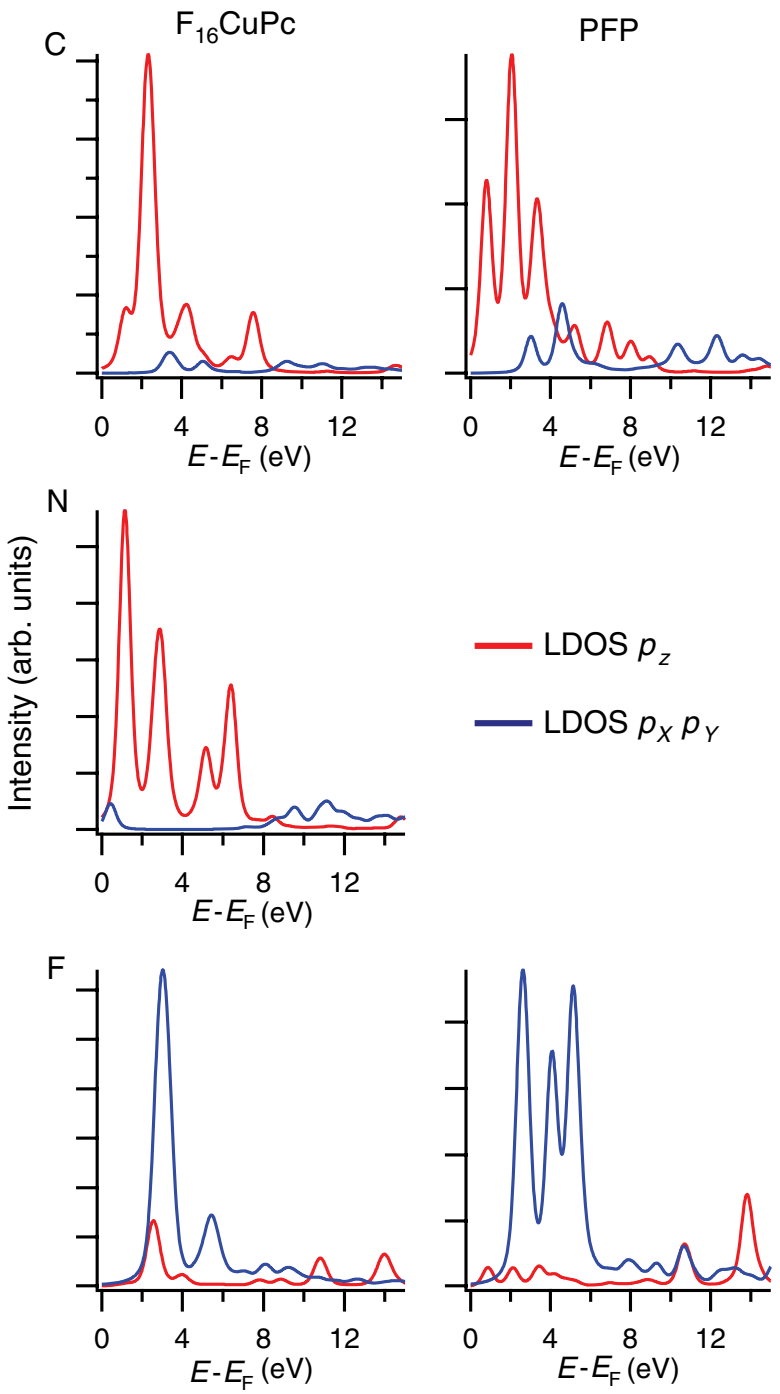

FIG. 3. (Color online) LDOS from unoccupied $p_{z}$ or $p_{x}-p_{y}$ orbitals projected onto the $\mathrm{F}_{16} \mathrm{CuPc}$ and PFP C, N, and $\mathrm{F}$ atoms, respectively. These correspond to the final states for the electron transitions of our subsequently measured NEXAFS spectra.

former corresponds to $\sigma^{*}$ orbitals and does not dominate the LDOS until $\sim 10 \mathrm{eV}$ above the Fermi level. Such a scenario is optimum to obtain reliable, quantitative structural information about molecular orientations from the analysis of NEXAFS intensities as a function of the angle between substrate surface and the X-ray polarization vector. This is because of the possibility to focus on NEXAFS resonances that correspond purely to $\pi^{*}$ orbitals (at the lowest energies). As soon as contributions from both $\pi^{*}$ and $\sigma^{*}$ orbitals overlap in measured NEXAFS intensity at a given energy, the extraction of structural information from such analysis is hampered.

The projected LDOS on $\mathrm{N}$ atoms in $\mathrm{F}_{16} \mathrm{CuPc}$ close to the Fermi level shows contributions from unoccupied $p_{z}$ and $p_{x}-p_{y}$ states overlapping in energy. Instead, at higher energies in the range from 2 to $7 \mathrm{eV}$ above the Fermi level, only $p_{z}$ states are present. Therefore, instead of using the very first resonance for the analysis of angle-dependent NEXAFS measurements, as is common practice, it is the higher energy resonances involving these $p_{z}$ states that should optimally be used. However, regardless of the minor contribution from $p_{x}-p_{y}$ states at low energies, it is still the $p_{z}$ states that dominate the LDOS. This is the expected scenario, since $\pi$ and $\pi^{*}$ orbitals are commonly those closest to the Fermi level in organic semiconductors. Instead, as shown in the lower panels of Fig. 3, the opposite scenario is found when focusing on the LDOS projected onto the F atoms, regardless of the molecule. While the lowest energy states are actually $p_{z}$, they overlap in energy to some extent with $p_{x}-p_{y}$ states centered at only slightly higher energies. But most strikingly, close to the Fermi level, the latter have a much higher LDOS, in contrast with the distribution on $\mathrm{C}$ and $\mathrm{N}$. As we will see below, this leads to the inversed linear dichroism observed in F $K$-edge NEXAFS spectra.

Figure 4 shows experimental NEXAFS spectra of the C, N, and $\mathrm{F} K$-edges of $\mathrm{F}_{16} \mathrm{CuPc}$ and PFP monolayers on $\mathrm{Au}(111)$.

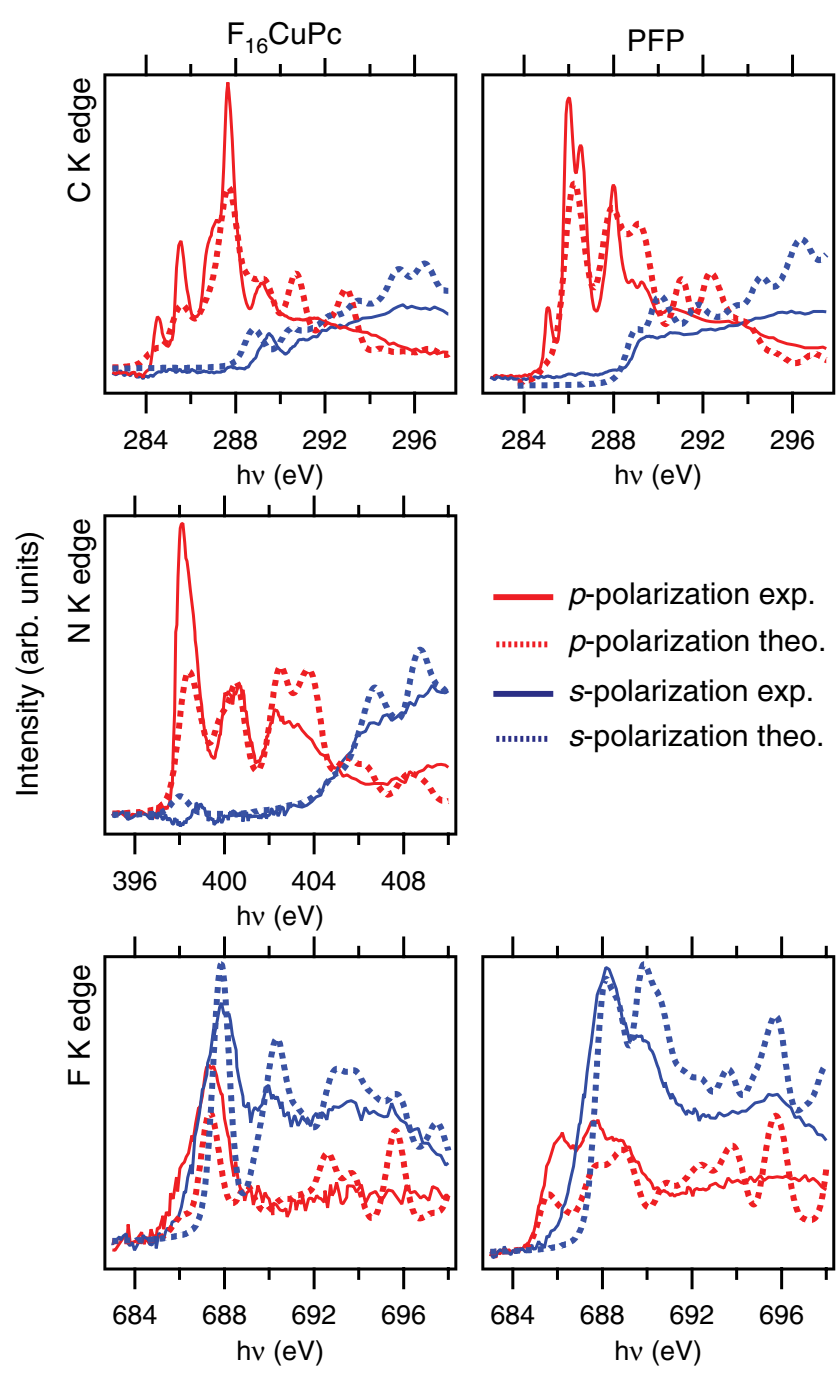

FIG. 4. (Color online) NEXAFS spectra from the $K$-edges of C, $\mathrm{N}$, and $\mathrm{F}$ in $\mathrm{F}_{16} \mathrm{CuPc}$ and PFP monolayers on $\mathrm{Au}(111)$ for $p$ - and $s$-polarizations. Solid lines show the experimental spectra, while the dotted lines depict the spectra calculated for gas-phase molecules. The latter are shifted to lower energies by the following amounts: $\mathrm{C}_{\mathrm{CuPc}}=-1.5 \mathrm{eV}, \mathrm{C}_{\mathrm{PFP}}=-1.3 \mathrm{eV}, \mathrm{F}_{\mathrm{CuPc}}=-2.2 \mathrm{eV}, \mathrm{F}_{\mathrm{PFP}}=-2.0 \mathrm{eV}$, and $\mathrm{N}_{\mathrm{CuPc}}=-1.1 \mathrm{eV}$. 
The spectra are taken under both $p$ - and $s$-polarization. Both molecules are known to adsorb with their molecular plane parallel to the $\mathrm{Au}(111)$ surface. Furthermore, the relatively weak molecule-substrate interactions and large moleculesubstrate distance has been shown to lead to undistorted molecular geometries. ${ }^{29}$ Under these circumstances, $p$ - and $s$-polarizations probe $\pi^{*}$ and $\sigma^{*}$ orbitals, respectively. For comparison with gas phase spectra, monolayers on a weakly interacting substrate as $\mathrm{Au}(111)$ appear as an optimum system. However, disregarding minor differences, the same inversed linear dichroism is found for monolayers on more reactive surfaces as $\mathrm{Cu}(111)$ or $\mathrm{Ag}(111)$ (see Supplemental Material ${ }^{30}$ ). As expected from the calculations in Fig. 3, the $\mathrm{C} K$-edge spectra of both $\mathrm{F}_{16} \mathrm{CuPc}$ and PFP show a pronounced linear dichroism. At the lowest energies we find strong $\pi^{*}$ resonances, observed solely under $p$-polarization, and an absolute absence of $\sigma^{*}$ resonances. Focusing now on the $\mathrm{F}_{16} \mathrm{CuPc} \mathrm{N}$ $K$-edge, the experimental observations agree again with the expectations based on the calculated LDOS, that is, we find the edge intensity dominated by a $\pi^{*}$ resonance but overlapping in energy with a considerable intensity from a $\sigma^{*}$ resonance. This model interpretation consistently explains also previous reports for the planar phases of $\mathrm{CuPc}$ on $\mathrm{Au}(110)$, where a residual $\sigma^{*}$-symmetry feature was reported at a $\mathrm{BE} \sim 0.4 \mathrm{eV}$ higher than the first $\pi^{*}$ LUMO resonance at $398.3 \mathrm{eV}$, and it was formerly attributed to substrate-induced rehybridization. ${ }^{32}$ Instead, higher-energy $\pi^{*}$ resonances between 399 and $405 \mathrm{eV}$ do not overlap in energy with $\sigma^{*}$ contributions and are therefore more recommendable for the analysis of angle-dependent measurements.

Finally, the F $K$-edge shows a completely different behavior. On both $\mathrm{F}_{16} \mathrm{CuPc}$ and PFP, the edge resonances correspond to $\pi^{*}$ orbitals but overlap in energy with $\sigma^{*}$ orbitals centered at only slightly higher energies and, most interestingly, with a much larger intensity. This leads to an inversed linear dichroism in angle-dependent NEXAFS spectra as compared to $\mathrm{C}$ or $\mathrm{N}$ (the latter only for $\mathrm{F}_{16} \mathrm{CuPc}$ ). That is, while for the $\mathrm{F}$ edge, the strongest NEXAFS intensity at low energies is observed under $s$-polarization as a result of the larger LDOS of $p_{x}-p_{y}$ states, for the $\mathrm{C}$ or $\mathrm{N}$ edge, the strongest intensity in the spectra is found under $p$-polarization due to the larger LDOS of $p_{z}$ states on those atoms. The linear dichroism at the $\mathrm{F}$ edge is, however, in line with that found at the $\mathrm{Cu} L$ edge of $\mathrm{F}_{16} \mathrm{CuPc}^{31}$ In that case, the main peak is related to a transition from the copper core $\mathrm{Cu} 2 p_{x, y}$ localized orbital into the single lowest unoccupied molecular orbital (SUMO), mainly of $\mathrm{Cu} 3 d_{x 2-y 2}$ character. The transition matrix is maximal when the electric field is parallel to the molecular plane and minimal when perpendicular. No other excitations with differently oriented transition matrices overlap in energy, making it perfectly suited for determination of molecular orientations from angle-dependent NEXAFS measurements. ${ }^{32}$ In contrast, the $\mathrm{F} K$-edge is not suited for such analysis and can lead to erroneous conclusions. As explained above, the analysis is based on the well-defined angular dependence of transition matrices and can therefore only be used if the NEXAFS intensity analyzed stems from one or also more transition matrices, as long as they share the same orientation. In the case of the $\mathrm{F} K$-edge, throughout the whole energy range, there are unoccupied $p_{z}$ and $p_{x}-p_{y}$ states that overlap in energy and have correspondingly orthogonal transition matrices (Fig. 3). The highly convoluted intensity from orthogonally oriented transition matrices (and therefore with different dependence on the polarization angle), in turn, impedes correct determination of molecular orientations from the analysis of measured intensity in polarization-dependent F $K$-edge NEXAFS spectra. Okudaira et al., for example, noticed the inversed linear dichroism in the $\mathrm{F} K$-edge as compared to the $\mathrm{C} K$-edge of $\mathrm{F}_{16} \mathrm{CuPc}^{33}$ However, in their analysis of the polarization-dependent measurements, they did not realize the overlapping contributions related to slightly shifted $\pi^{*}$ and $\sigma^{*}$ orbitals. Instead, they assumed the intensity to stem entirely from a pure $\sigma^{*}$ resonance, and although the conclusions they reached were in accordance with the results from $\mathrm{C} K$-edge NEXAFS spectra, this can only have been error cancellation.

We have shown how the calculated density of states of unoccupied $p_{z}$ or $p_{x}-p_{y}$ orbitals projected onto the $\mathrm{F}_{16} \mathrm{CuPc}$ and PFP $\mathrm{C}, \mathrm{N}$, and $\mathrm{F}$ atoms provide a coherent picture and understanding of the experimentally measured angledependent NEXAFS spectra. Taking one step further, we have calculated theoretical NEXAFS spectra on undistorted, gas phase molecules and compared them to the measurements (Fig. 4). The calculated spectra were shifted down in energy to provide an optimum matching between theory and experiment. Such a shift, on the order of $1.3 \mathrm{eV}$, is typical in DFT calculations of NEXAFS spectra using the TPA approximation. ${ }^{34}$ After this rigid energy shift, a strikingly good agreement is observed between theory and experiment. An unambiguous correspondence between the spectral features in experiment and calculations provides detailed understanding of the electronic structure of our molecules. It also confirms the different linear dichroism behavior observed at the $\mathrm{F} K$-edge, as opposed to the $K$-edges of $\mathrm{C}$ and $\mathrm{N}$, and thereby evidences the different symmetry of the orbitals with strong weight on the $\mathrm{F}$ atoms with respect to those on the main molecular backbone.

\section{CONCLUSION}

In conclusion, we provide a detailed understanding of the energies and symmetry of the unoccupied molecular orbitals of PFP and $\mathrm{F}_{16} \mathrm{CuPc}$. Such information is critical for studies of their molecular arrangement and orientation in thin films or monolayers by NEXAFS. We have shown the inconvenience of using the $\mathrm{F} K$ edge for this aim. Regardless of the molecule, it shows an inversed linear dichroism as compared to the commonly measured $\mathrm{C} K$-edge, but it is the significant overlap in energy of $\pi^{*}$ and $\sigma^{*}$ orbitals that hampers the analysis of angle-dependent NEXAFS measurements at this edge.

\section{ACKNOWLEDGMENTS}

J.E.O. and A.R. acknowledge funding from the Spanish MEC through Grants No. FIS2011-65702-C02-01, No. MAT2010-21156-C03-01, and No. PIB2010US-00652, and from the Basque Government through Grants No. IT-257-07 and No. IT-319-07. A.R. additionally acknowledges that financial support was provided by ACI-Promociona Grant No. ACI2009-1036 and the European Research Council 
Advanced Grant DYNamo (ERC-2010-AdG, Proposal No. 267374). A.S. acknowledges the support of the Research Funds of the University of Helsinki and the Academy of Finland through Contract No. 1127462, Centers of Excellence
Program, and the National Graduate School in Materials Physics. J.M.G.L. acknowledges support from The Lundbeck Foundation's Center for Atomic-Scale Materials Design and the Danish Center for Scientific Computing. *d_g_oteyza@ehu.es

${ }^{1}$ N. Koch, Chem. Phys. Chem. 8, 1438 (2007).

${ }^{2}$ C. R. Newman, C. D. Frisbie, D. A. da Silva Filho, J.-L. Bredas, P. C. Ewbank, and K. R. Mann, Chem. Mater. 16, 4436 (2004).

${ }^{3}$ S. Duhm, G. Heimel, I. Salzmann, H. Glowatzki, R. L. Johnson, A. Vollmer, J. P. Rabe, and N. Koch, Nat. Mater. 7, 326 (2008).

${ }^{4}$ W. Chen, D.-C. Qi, H. Huang, X. Gao, and A. T. S. Wee, Adv. Funct. Mater. 21, 410 (2011).

${ }^{5}$ J. Stohr, NEXAFS Spectroscopy (Springer, Heidelberg, 2003).

${ }^{6}$ L. Cao, Y. Wang, J. Zhong, Y. Han, W. Zhang, X. Yu, F. Xu, D.-C. Qi, and A. T. S. Wee, J. Phys. Chem. C 115, 24880 (2011).

${ }^{7}$ M. Marks, C. Schmidt, C. H. Schwalb, T. Breuer, G. Witte, and U. Hofer, J. Phys. Chem. C 116, 1904 (2012).

${ }^{8}$ S. Sohnchen, S. Lukas, and G. Witte, J. Chem. Phys. 121, 525 (2004).

${ }^{9}$ M. Piantek, J. Miguel, A. Kruger, C. Navio, M. Bernien, D. K. Ball, K. Hermann, and W. Kuch, J. Phys. Chem. C 113, 20307 (2009).

${ }^{10}$ R. Schmidt, S. Hagen, D. Brete, R. Carley, C. Gahl, J. Dokic, P. Saalfrank, S. Hecht, P. Tegeder, and M. Weinelt, Phys. Chem. Chem. Phys. 12, 4488 (2010).

${ }^{11}$ D. G. de Oteyza, I. Silanes, M. Ruiz-Oses, E. Barrena, B. P. Doyle, A. Arnau, H. Dosch, Y. Wakayama, and J. E. Ortega, Adv. Funct. Mater. 19, 259 (2009).

${ }^{12}$ M. Ruiz-Oses, D. G. de Oteyza, I. Fernandez-Torrente, N. Gonzalez-Lakunza, P. M. Schmidt-Weber, T. Kampen, K. Horn, A. Gourdon, A. Arnau, and J. E. Ortega, Chem. Phys. Chem. 10, 896 (2009).

${ }^{13}$ M. L. Tang and Z. Bao, Chem. Mater. 23, 446 (2011).

${ }^{14}$ D. G. de Oteyza, E. Barrena, Y. Zhang, T. N. Krauss, A. Turak, A. Vorobiev, and H. Dosch, J. Phys. Chem. C 113, 4234 (2009).

${ }^{15}$ As calculated from the thin film crystal-structure reported in I. Salzmann, S. Duhm, G. Heimel, J. P. Rabe, N. Koch, M. Oehzelt, Y. Sakamoto, and T. Suzuki, Langmuir 24, 7294 (2008).

${ }^{16} \mathrm{See}$ Supplemental Material at http://link.aps.org/supplemental/ 10.1103/PhysRevB.86.075469 for NEXAFS data of $\mathrm{F}_{16} \mathrm{CuPc}$ and PFP monolayers in a wider energy range, including the postedge intensities.

${ }^{17}$ L. Floreano, G. Naletto, D. Gvetko, R. Gotter, M. Malvezzi, L. Marassi, A. Morgante, A. Santaniello, A. Verdini, F. Tommasini, and G. Tondello, Rev. Sci. Instrum. 70, 3855 (1999).
${ }^{18}$ J. C. Fuggle and N. Martensson, J. Electron Spectrosc. Relat. Phenom. 21, 275 (1980).

${ }^{19}$ K. Hermann, L. G. M. Pettersson, M. E. Casida, C. Daul, A. Goursot, A. Koester, E. Proynov, A. St-Amant, and D. R. Salahub, Contributing authors: V. Carravetta, H. Duarte, C. Friedrich, N. Godbout, J. Guan, C. Jamorski, M. Leboeuf, M. Leetmaa, M. Nyberg, S. Patchkovskii, L. Pedocchi, F. Sim, L. Triguero, and A. Vela, StoBe-deMon version 3.0 (2009), http://www.fhi-berlin.mpg.de/KHsoftware/ StoBe/index.html.

${ }^{20}$ S. H. Vosko, L. Wilk, and M. Nusair, Can. J. Phys. 58, 1200 (1980).

${ }^{21}$ L. Triguero, L. G. M. Pettersson, and H. Ågren, Phys. Rev. B 58, 8097 (1998).

${ }^{22}$ All basis sets are available at http://www.demon-software.com/ public_html/download.html\#basis, 3rd January 2012.

${ }^{23}$ S. Huzinaga, L. Seijo, Z. Barandiarán, and M. Klobukowski, J. Chem. Phys. 86, 2132 (1987).

${ }^{24}$ J. P. Perdew, K. Burke, and M. Ernzerhof, Phys. Rev. Lett. 77, 3865 (1996).

${ }^{25}$ H. Peisert, M. Knupfer, T. Schwieger, G. G. Fuentes, D. Olligs, J. Fink, and Th. Schmidt, J. Appl. Phys. 93, 9683 (2003).

${ }^{26}$ H. Peisert, M. Knupfer, and J. Fink, Surf. Sci. 515, 491 (2002).

${ }^{27}$ I. G. Hill, A. Kahn, Z. G. Soos, and R. A. Pascal, Chem. Phys. Lett. 327, 181 (2000).

${ }^{28}$ J. J. Olivero and R. L. Longbothum, J. Quant. Spectrosc. Radiat. Transfer 17, 233 (1977).

${ }^{29}$ D. G. de Oteyza, A. El-Sayed, J. M. Garcia-Lastra, E. Goiri, T. N. Krauss, A. Turak, E. Barrena, H. Dosch, J. Zegenhagen, A. Rubio, Y. Wakayama, and J. E. Ortega, J. Chem. Phys. 133, 214703 (2010).

${ }^{30}$ See Supplemental Material at http://link.aps.org/supplemental/ 10.1103/PhysRevB.86.075469 for NEXAFS data of $\mathrm{F}_{16} \mathrm{CuPc}$ and PFP monolayers on $\mathrm{Cu}(111)$ and $\mathrm{Ag}(111)$, respectively.

${ }^{31}$ S. Carniato, Y. Luo, and H. Agren, Phys. Rev. B 63, 085105 (2001).

${ }^{32}$ L. Floreano, A. Cossaro, R. Gotter, A. Verdini, G. Bavdek, F. Evangelista, A. Ruocco, A. Morgante, and D. Cvetko, J. Phys. Chem. C 112, 10794 (2008).

${ }^{33}$ K. K. Okudaira, H. Setoyama, H. Yagi, K. Mase, S. Kera, A. Kahn, and N. Ueno, J. Electron Spectrosc. Relat. Phenom. 137-140, 137 (2004).

${ }^{34}$ A. Sakko, M. Hakala, J. A. Soininen, and K. Hämäläinen, Phys. Rev. B 76, 205115 (2007). 